\title{
Revisión analítica del modelo de aceptación de la tecnología. El cambio tecnológico
}

\author{
Cristóbal Torres Albero \\ Universidad Autónoma de Madrid \\ cristobal.torres@uam.es \\ José Manuel Robles \\ Stefano de Marco \\ Mirko Antino \\ Universidad Complutense de Madrid \\ jmrobles@ccee.ucm.es; s.demarco@cps.ucm.es; m.antino@psi.ucm.es
}

Recepción: 22-06-2015

Aceptación: 25-06-2016

\section{Resumen}

El modelo de aceptación de la tecnología (TAM) es uno de los enfoques individualistas metodológicos más relevantes en el estudio del uso de Internet. El presente trabajo constituye una revisión del debate sobre el peso de las variables psicológicas y sociales en este modelo. Nuestra tesis es que, a medida que Internet va penetrando socialmente, las variables psicológicas, como las creencias subjetivas sobre las posibilidades del medio, van cediendo protagonismo. Por el contrario, las variables sociales se transforman en los mejores predictores del uso de Internet, ya que son los estímulos y los inhibidores más importantes para la adopción de este comportamiento. En esta línea, los estudios empíricos muestran que la capacidad predictiva de las variables psicológicas es ahora menor que la de las variables sociales. En el presente trabajo, mostramos empíricamente dicha circunstancia y defendemos el papel de un modelo individualista metodológico de corte sociológico, basado en la preponderancia de las variables sociológicas sobre las psicológicas para explicar el uso de Internet.

Palabras clave: modelo TAM; brecha digital; Internet; cambio social; sociedad de la información y del conocimiento 


\section{Abstract. Analytical Review of the Technology Acceptance Model: Technological Change}

The Technology Acceptance Model (TAM) is one of the most important methodological individualist approaches in the study of Internet use. This paper reviews the debate on the weight of psychological and social variables in this model. Our thesis is that, as the Internet is penetrating society and becoming increasingly popular, psychological variables such as subjective beliefs about the possibilities of technology are gaining prominence. By contrast, social variables are becoming better predictors of Internet use as they are stimuli and inhibitors leading to the adoption of this behavior. For this reason, empirical studies repeatedly show with different measurement tools that the predictive power of psychological variables is now lower than that of social variables. In this paper, we demonstrate this empirically and defend the role of a methodological individualist model from a sociological approach based more on sociological than psychological variables for explaining the use of the Internet.

Keywords: Technology Acceptance Model; TAM; digital divide; Internet; social change; information and knowledge society

\section{Sumario}

1. Introducción

2. Marco teórico

3. Metodología

4. Resultados
5. Discusión

6. Conclusión

Referencias bibliográficas

Anexo 1

\section{Introducción ${ }^{1}$}

En un trabajo anterior (Torres et al., 2011), analizábamos los motivos por los que unos ciudadanos y no otros deciden comenzar a utilizar Internet. Nos basábamos en uno de los modelos individualistas metodológicos más utilizados en la literatura técnica: el de aceptación de la tecnología (TAM, en sus siglas en inglés). Este modelo puede considerarse bajo el paraguas del individualismo metodológico, en tanto que se centra en el comportamiento de los individuos para la explicación social. Dicha explicación se basaba en el análisis de las creencias y de las actitudes de los sujetos, así como en sus oportunidades y recursos (Davis, 1986 y 1989). Sin embargo, la versión original del TAM depositaba gran parte del peso del modelo explicativo sobre dos tipos de creencias: la percepción de la utilidad de la tecnología y la facilidad percibida de uso de la tecnología. Nuestro artículo ponderaba, por el contario, el peso de las variables sociodemográficas y socioeconómicas, en un intento por completar la capacidad explicativa del modelo original, marcadamente psicológico. Para ello, tratamos de mostrar la relevancia de las variables edad y nivel de estudios para predecir el uso de Internet en el marco de este modelo. Se trataba, por lo

1. Este artículo se enmarca dentro de los proyectos CSO2009-13424 y CSO2012-35688, del Ministerio de Economía y Competitividad del Gobierno de España. 
tanto, de un esfuerzo para traer las bondades del TAM a un escenario propio de la sociología. El artículo suscitó cierto interés y generó un interesante debate (Torres et al., 2012)

Algún tiempo después, hemos tenido la oportunidad de diseñar una nueva encuesta cuyos indicadores fueron pensados teniendo en cuenta, sino todos los elementos del debate actual sobre el modelo de aceptación de la tecnología, sí los requerimientos más importantes exigidos tanto por la literatura como por nuestros comentaristas. El presente artículo ha sido realizado para reforzar nuestra defensa de la importancia de un modelo TAM sociológico, mantener abierto el debate iniciado sobre este tema de trabajo y, en definitiva, reclamar la utilidad de dicho tipo de enfoques epistemológicos para el estudio de la sociología de la tecnología.

Así, haciendo uso de la nueva encuesta, tratamos de corroborar, en primer lugar, el modelo clásico de la teoría de aceptación de la tecnología. Según este modelo, las variables psicológicas determinan el uso de Internet, mientras que las variables contextuales, en nuestro caso de carácter sociodemográfico (Torres et al., 2011), están mediadas por dichas variables psicológicas (López-Bonilla y López-Bonilla, 2012) y no se relacionan directamente con la variable dependiente. Para corroborar este modelo clásico, hemos realizado un path analysis que nos permite comprobar la fiabilidad y la validez del citado modelo. Los resultados de este análisis nos mostrarán las limitaciones del modelo clásico para predecir el uso de Internet, tomando como un estudio de caso los datos actuales en España.

En segundo lugar, tratamos de mostrar la capacidad explicativa de un modelo TAM sociológico. Para ello, introducimos, junto a las variables sociodemográficas utilizadas en nuestro estudio anterior, tres nuevos grupos de variables relacionadas con las habilidades digitales, el tipo de conexión a Internet y la tecnología de conexión. Todas ellas representan un lugar común en el estudio de la brecha digital y su uso está justificado por la literatura sobre este tema (Van Dijk, 2005; DiMaggio et al., 2001). Este análisis nos permitirá mostrar el peso de las variables contextuales y sociodemográficas y, al mismo tiempo, la pérdida de valor de las variables psicológicas en el estudio del uso de Internet. Este trabajo completa y refuerza los resultados obtenidos en nuestro artículo de 2011 y permite seguir discutiendo sobre el desarrollo y la mejora del modelo TAM.

Para este análisis, también hemos mejorado la operacionalización de nuestra variable dependiente. Así, hemos tratado de superar la lectura dicotómica que suponía una variable diseñada para medir el número de ciudadanos que utilizaban o no Internet. Tanto la literatura técnica (Van Dijk, 2005) como los comentaristas de nuestros trabajos (López-Bonilla y López-Bonilla, 2012) han advertido sobre la simplificación que implica este tipo de variables. Por dicho motivo, hemos construido una variable compleja que mide, a través de una escala, el grado en que un ciudadano es usuario de Internet en función del número y del tipo de usos que otorga a esta tecnología, así como de la frecuencia de realización. Así pues, tenemos en un extremo a los ciudadanos que usan 
Internet todos los días para un conjunto plural y heterogéneo de actividades y, en el otro extremo, a los que usan Internet de forma muy esporádica y únicamente para actividades concretas y simples.

En este trabajo, tratamos de explicar los resultados empíricos obtenidos desde el concepto de cambio tecnológico (Norris, 2001). Desde nuestro punto de vista, las variables psicológicas que definen el núcleo central del TAM son muy relevantes para predecir el uso de Internet. La literatura lo ha mostrado sobradamente (Davis, 1986 y 1989). Sin embargo, nuestra hipótesis es que estas variables van perdiendo protagonismo a medida que avanza el proceso de penetración del uso de Internet. Cuando esto sucede, las creencias favorables hacia las posibilidades y la facilidad de uso de las tecnologías se van generalizando entre los ciudadanos y dejan de ser variables discriminatorias. En este nuevo contexto tecnológico, la importancia de las variables sociales, como el nivel de estudios o la edad, aumentan. Estas variables se transforman en favorecedoras o limitadoras del uso de tecnologías y, más importante, en inhibidoras o estímuladoras para su uso frecuente y avanzado. Las habilidades digitales, así como la disponibilidad de recursos materiales para conectase a Internet, también se transforman en elementos clave para el uso de esta tecnología, una vez superada la fase en la que los ciudadanos deben comprender su utilidad práctica. Esta interpretación de nuestros resultados nos permite vincular una explicación individualista metodológica, en nuestro caso, relacionando recursos individuales y procesos sociales más generales con las características de la tecnología y los valores sociales. Intentamos así cumplir con una de las exigencias básicas del enfoque analítico de la sociología (Noguera, 2010). Igualmente, tratamos de evitar la lectura determinista, según la cual el desarrollo de las tecnologías impacta sobre los valores, las actitudes y las creencias de los ciudadanos, y esto, a su vez, genera cambio social (Levy, 2007).

\section{Marco teórico}

\subsection{Algunas claves sobre nuestra propuesta de estudio del modelo de aceptación de la tecnología}

El modelo de aceptación de la tecnología ${ }^{2}$ pivota en torno a tres ejes fundamentales: la utilidad percibida de la tecnología (UP), la facilidad de uso de la tecnología (FUP) y las actitudes sobre la tecnología (Venkatesh et al., 2003). Los dos primeros constituyen dos tipos de creencias y el tercero, un tipo de disposición actitudinal. Los autores clásicos de la disciplina definen la UP como

2. En este apartado, realizaremos un breve resumen sobre los aspectos fundamentales del modelo, así como sobre su desarrollo temporal. En nuestro artículo de 2011, así como en la réplica de López-Bonilla y López-Bonilla (2012), se presenta una formulación teórica más elaborada. Este resumen implica no explicitar las diferencias entre los distintos modelos TAM, TAM 2, etc. Desde nuestro punto de vista, es más relevante centrarse en los elementos añadidos para este segundo tratamiento del problema, sin descuidar, naturalmente, la explicación de los rudimentos del modelo. 
la probabilidad subjetiva de que el uso de una tecnología mejore el rendimiento de las actividades que realiza una persona. Es decir, se refiere a la percepción general sobre las ventajas que reporta el uso de un instrumento tecnológico. Por su parte, la FUP se refiere al grado de dificultad atribuida al uso de una tecnología. En otras palabras, la FPU advierte sobre en qué medida una tecnología es percibida como un coste para la acción (Davis, 1989).

Este tipo de creencias presenta, al menos en el modelo original, una fuerte correlación con la actitud de una persona hacia las tecnologías (Davis et al., 1989). Dicha actitud es, a su vez, el mejor indicador para predecir la disposición y, finalmente, su uso (Mathieson, 1991). Sin embargo, tal y como advierten López-Bonilla y López-Bonilla (2012), a lo largo del desarrollo del estudio sobre el TAM, se ha mostrado la escasa incidencia de las actitudes en este modelo y, por lo tanto, se ha descartado su uso en TAM más avanzados (Davis et al., 1992; Venkatesh y Davis, 2000).

Otro de los ámbitos en los que el TAM más ha adelantado está relacionado con las variables contextuales que afectan al modelo. Desde un punto de vista psicológico, se mostró la estrecha relación existente entre los lazos psicológicos (psycological attachment) y la actitud favorable hacia el uso de las tecnologías (Davis et al., 1989; Lin y Bhattacherjee, 2009). Desde este punto de vista, los incentivos individuales, tanto negativos como positivos, asociados a la utilización de una tecnología determinan en gran medida la disposición de una persona para usarla o no. Otro factor contextual se refiere a la medida en que el uso de una tecnología permite al usuario desarrollar lazos de identificación con un colectivo o con un grupo de personas. Por último, las actitudes hacia una tecnología están también definidas por el grado de congruencia y consistencia entre esa tecnología y el resto de valores y actitudes que definen la personalidad del sujeto (Malhotra y Galleta, 1999).

En el estudio sobre los factores contextuales que afectan al modelo TAM, existe otra importante línea de investigación relacionada con la Innovation Difussion Theory (IDT). Desde este punto de vista, los investigadores han tratado de comprender hasta qué punto la percepción sobre los recursos materiales disponibles afectan a la decisión de usar una tecnología (Taylor y Todd, 1995). En este sentido, se ha tratado de estimar la influencia de las condiciones tecnológicas materiales sobre la percepción de la utilidad de las tecnologías. Sin embargo, es interesante advertir que, hasta donde sabemos, la literatura no ha utilizado como variables contextuales materiales el tipo de conexión a Internet, ni tampoco los lugares de uso de esta tecnología. La disponibilidad de dichos recursos se ha transformado en un predictor muy importante del uso de Internet. Desde nuestro punto de vista, se trata de un elemento fundamental, ya que la literatura que trata sobre la brecha digital ha mostrado la estrecha relación existente entre estas dos variables y el uso de Internet, especialmente el uso avanzado de esta tecnología (Van Dijk, 2005).

La literatura sobre la brecha digital ha señalado, igualmente, que las habilidades digitales (o digital skills) constituyen un factor fundamental para predecir el uso de Internet (Hargittai, 2008 y 2010). Sin embargo, los especialistas en 
el TAM no han incorporado esta variable a su esquema de análisis. Tal y como sostiene Quintanilla (2005), las habilidades requeridas para hacer uso de una tecnología pueden ser genéricas o específicas. Si una determinada tecnología demanda habilidades genéricas, es esperable que estas no se transformen en una barrera para su utilización. Sin embargo, si, como es el caso de Internet, las habilidades demandadas son específicas, poseerlas o no es una cuestión a tener en cuenta.

Sin embargo, el modelo TAM sí ha tenido presente el papel de variables contextuales referidas a los recursos individuales, como el nivel de estudios, la edad o el género (Venkatesh et al., 2003; Lynott y McCandless, 2000; Morris y Venkatesh, 2000). Torres et al. (2011) mostraron cómo, entre estas variables, la edad y el nivel de estudios eran las que mayor capacidad predictiva mostraban en el marco del modelo TAM. Este y otros resultados similares suponen un primer paso para un TAM de corte sociológico. Sin embargo, aún queda mucho por avanzar en esta dirección, ya que, por ejemplo, aún no sabemos cómo se relacionan las variables psicológicas del TAM con otras variables sociales, como los valores postmaterialistas, y contextuales, como las condiciones materiales de acceso o las habilidades digitales de los ciudadanos.

El desarrollo experimentado por el modelo TAM a lo largo de las últimas décadas muestra el esfuerzo por incluir variables contextuales que mejoren la capacidad del modelo. Sin embargo, como hemos visto más arriba, existen variables que la literatura sobre la brecha digital ha mostrado que son fundamentales para predecir el uso de Internet. Desde nuestro punto de vista, es importante establecer un diálogo con estos campos de estudio para tratar de ofrecer mejoras en el modelo TAM. Nuestra propuesta, tal y como veremos en el apartado metodológico, va en esta dirección. Sin embargo, antes de pasar a dicho apartado, nos gustaría reflexionar sobre la forma en la que penetra el uso de Internet en una determinada población. Esto nos servirá como marco teórico general con el que evaluar la fortaleza de nuestra hipótesis.

\subsection{Normalización versus estratificación. Un modelo sociológico para el estudio de la difusión del uso de Internet}

El estudio del cambio tecnológico es una de las líneas clásicas de las ciencias sociales (Gómez et al., 1992). En su conocido libro sobre el cambio tecnológico Elster (1990) organiza las explicaciones sobre el mismo en cuatro grandes grupos: teoría económica clásica, teoría marxista, enfoque evolucionista y enfoque constructivista. Pese a sus diferencias, estos enfoques analizan el proceso de desarrollo tecnológico a través de un prisma dinámico. Es decir, señalan los mecanismos mediante los cuales una tecnología pasa de ser una herramienta marginal a ser una tecnología social, económica y políticamente aceptada. Los especialistas en el estudio de la brecha digital y la desigualad digital han desarrollado distintos conceptos para describir, también desde un punto de vista dinámico, el proceso de penetración de las tecnologías de la información y la comunicación. En este y en otros trabajos (Robles et al., 2010; Robles y Torres, 
2012; Torres et al., 2013), nos hemos centrado en dos de ellos, normalización y estratificación, para mostrar la importancia del segundo.

El concepto de estratificación comparte, con la nueva sociología de la tecnología (Aibar, 1996), un esfuerzo por escapar, en la misma medida, del determinismo tecnológico y del determinismo sociológico ${ }^{3}$. Igualmente, trata de enfatizar los procesos de configuración social de la tecnología (Mackenzie y Wajcman, 1999). Esto es, defiende la convicción de que la relación entre tecnología y sociedad debe explicarse atendiendo a la forma en que factores sociales de distinto orden influyen sobre el uso y las características de las herramientas tecnológicas.

La idea de normalización surge para describir un proceso de evolución de la penetración de Internet, en el que las diferencias de partida entre grupos sociales cuyas actitudes y creencias hacia las tecnologías son más o menos favorables, tienden a descender como consecuencia de las medidas públicas y privadas de reducción de la brecha digital (Norris, 2001). Se trata de una perspectiva optimista sobre cómo evoluciona el uso de Internet, así como sobre la capacidad institucional para modificar las creencias de los ciudadanos sobre las posibilidades del medio. Es también una perspectiva determinista, en la medida en que considera que la provisión de acceso y la facilitación del uso de Internet terminarán con las desigualdades tecnológicas.

Frente a la tesis de la normalización, la perspectiva de la estratificación parte de un punto de vista algo menos optimista. Supone que los distintos grupos sociales tienen diferentes niveles máximos de penetración del uso de Internet. Estos niveles máximos están definidos por las propias características del grupo social (sus valores y creencias), por la utilidad subjetiva que dicho grupo hace de Internet, así como de las propias herramientas y servicios que ofrecen. Algunos especialistas han alertado sobre la influencia que tienen sobre la percepción de la utilidad de Internet las características del propio medio. Se considera así que la red se ajusta mejor a los intereses y a las expectativas de unos grupos sociales, mientras que se aleja de los objetivos vitales de ciudadanos pertenecientes a otros grupos sociales (Norris, 2001).

Desde este punto de vista, no es posible alcanzar una inclusión digital universal, sino, a lo sumo, aumentar el porcentaje de internautas entre los grupos sociales menos permeables al uso de esta herramienta. Esto implicaría, en cualquier caso, que el desarrollo de la sociedad de la información y el conocimiento va a reflejar, pese a los esfuerzos institucionales, las desigualdades estructurales existentes en una comunidad determinada.

En varios estudios anteriores, hemos mostrado la existencia en España de un proceso de estratificación (Robles y Torres, 2012; Torres et al., 2013). Esto

3. En términos generales, el determinismo tecnológico considera, en primer lugar, que el desarrollo tecnológico es autónomo y, en segundo lugar, que las tecnologías son el principal factor de cambio social, puesto que afectan a las creencias, a los valores y a las normas sociales. Por su parte, el determinismo social supone que el hecho tecnológico pierde su idiosincrasia en el mar de relaciones sociales. Es decir, la tecnología no es más que una construcción social. 
significa que el desarrollo de la sociedad de la información y el conocimiento se está produciendo en España en dos escenarios distintos. Por una parte, los grupos poblacionales con más formación (universitarios y personas con estudios medios) que o bien han alcanzado el nivel de saturación o bien están experimentando un crecimiento en el número de internautas que hace esperable que alcancen dicho nivel máximo de saturación. Por otro lado, encontramos los grupos poblacionales con menos estudios (estudios elementales o sin estudios terminados), entre los que no es predecible un nivel de saturación cercano al ciento por ciento. Esta circunstancia también se reproduce según las variables estatus socioeconómico y edad. En definitiva, el desarrollo de la sociedad de la información y el conocimiento en España está generando un proceso de desigualdad tecnológica que puede ser dependiente de la conjunción entre los valores y las creencias de los distintos grupos sociales y las características del medio.

Así, la penetración del uso de Internet en una determinada población se produce, en un primer momento, entre los grupos cuya percepción de la utilidad y la facilidad de uso de Internet es mayor. Sin embargo, aquellos grupos entre los que dicha percepción de Internet no es tan común quedan, al menos en un principio, al margen del proceso de penetración social de Internet. Sin embargo, y esta es la idea clave, incluso cuando estos últimos grupos comienzan a incorporar el uso de Internet de forma más o menos sistemática, cuentan con un nivel de saturación que no alcanza el cien por cien de la población. Esto se produce, tal y como explica el concepto de estratificación, porque las desigualdades digitales reproducen las desigualdades tradicionales. Es decir, son las desigualdades educativas, generacionales o económicas las que determinan el máximo de penetración del uso de una determinada tecnología. Nuestra interpretación es que esta es la razón por la que, al inicio del proceso de extensión del uso de Internet, las variables actitudinales son fundamentales para predecir el uso de esta tecnología. Sin embargo, a medida que este proceso va avanzando, son las variables sociales las que se posicionan como límites tecnológicos.

Consideramos fundamental analizar las posibilidades del modelo TAM desde el prisma del cambio tecnológico y el concepto de estratificación. Gracias a estos enfoques, sabemos que las preferencias y las opiniones sobre las tecnologías en general, y sobre Internet en particular, no son estáticas. Así, desde el punto de vista de la estratificación, sabemos que las preferencias y los comportamientos relacionados con el uso de Internet dependen, en gran medida, del grupo social de pertenencia. Igualmente, sabemos que, incluso entre los grupos sociales menos sensibles a la incorporación de las tecnologías, las creencias evolucionan y cambian. Por último, también sabemos que esta evolución no garantiza que todos los ciudadanos sean, finalmente, usuarios de Internet. Esta perspectiva dinámica debe ayudarnos a entender el uso de Internet. El modelo TAM ha mostrado su capacidad para el estudio del uso de las tecnologías. Sin embargo, es crucial que tratemos de incorporar una perspectiva dinámica que nos permita comprender cómo se transforma el modelo en un proceso - el de la penetración del uso de Internet - de naturaleza dinámica. 


\section{Metodología}

Para cumplir con los objetivos empíricos de este trabajo, hemos procedido mediante un estudio en dos fases. En primer lugar, hemos implementado un path analysis que recoge las opciones teórico-metodológicas indicadas por López-Bonilla y López-Bonilla (2012) y que tienen un marcado enfoque psicológico. Desde este punto de vista, existiría un efecto directo de las variables psicológicas del TAM sobre el uso de Internet. Simultáneamente, dichas variables recibirían la influencia de las variables sociodemográficas clásicas del estudio de la brecha digital: estatus socioeconómico, nivel de estudios y edad (ver figura 1).

Los resultados de este modelo se han comparado con un modelo de regresión lineal múltiple "por pasos», cuya variable dependiente es, nuevamente, el uso de Internet. La implementación de un modelo por pasos ha permitido diferenciar el peso de cada uno de los bloques de variables independientes en la explicación de la varianza de la variable dependiente. En el primer paso, se han incluido las variables sociodemográficas, es decir, edad, nivel de estudios y estatus socioeconómico. En segundo lugar, se ha añadido otro conjunto de variables tecnológico-contextuales, es decir el tipo de conexión a Internet y la cantidad de lugares de conexión. El tercer paso incluye la variable habilidades digitales. Finalmente, se han introducido las dos variables psicológicas, facilidad de uso y utilidad percibida, además de una escala de medición de los valores postmaterialistas como variable de medición del cambio social actitudinal. Mediante este análisis, tratamos de conjurar las variables psicológicas clásicas del TAM con variables tradicionalmente utilizadas en el análisis sociológico del cambio social y tecnológico. Estas son las variables sociodemográficas y socioeconómicas, las del contexto tecnológico y una que se utiliza generalmente para analizar los procesos de cambio social más amplios, los valores postmaterialistas (Inglehart, 1991). En resumen, nuestro modelo incluye dos dimensiones para el análisis: una dimensión psicológica y otra dimensión sociológica.

La comparación entre los resultados obtenidos por los dos modelos, y entre los porcentajes de varianza explicada de los diferentes bloques, permitirá disipar más las dudas sobre qué modelo, psicológico o social, permite predecir en mayor medida el uso de Internet.

\subsection{Datos}

Se han utilizado los datos obtenidos en una encuesta representativa de ámbito nacional realizadas en la modalidad CATI a un total de 1.526 personas. La totalidad de entrevistas se estratificaron por la intersección de hábitat y comunidad autónoma, y se distribuyeron de manera proporcional a los totales de cada nivel de hábitat y región. Se aplicaron cuotas de sexo y edad a la unidad última que supone la persona entrevistada. Partiendo de los criterios del muestreo aleatorio simple, para un nivel de confianza del 95,5\% (que es el habitualmente adoptado) y en la hipótesis más desfavorable de máxima 
indeterminación $(p=q=50)$, el margen de error de los datos referidos al total de la muestra es de $\pm 2,6$.

Sin embargo, para el análisis de los datos aquí considerados, se ha optado por escoger solo a los internautas, es decir, los sujetos que habían accedido a Internet en los tres meses anteriores a la entrevista. De este modo, se ha alcanzado una muestra total de 1.046 personas ${ }^{4}$. Esta decisión se debe a la voluntad de superar la simple dicotomía entre usuario y no usuario, y así estudiar el efecto de determinadas variables sobre el cuánto y el cómo se utiliza Internet.

\subsection{Variables}

En base a esta decisión, se optó por construir una variable dependiente que incluyese la frecuencia de acceso a Internet en los últimos tres meses y la variedad de usos de Internet adoptados en este mismo periodo. Para medir la frecuencia de uso de Internet, se ha utilizado la misma pregunta que el Instituto Nacional de Estadística usa como filtro para discriminar entre usuarios de Internet y no usuarios de Internet en la Encuesta sobre equipamiento y uso de tecnologias de información y comunicación en los hogares. Esta pregunta ofrece a los encuestados las siguientes opciones: «Utiliza Internet todos los días», «Utiliza Internet una vez a la semana», "Ha utilizado Internet en el último mes», «Ha utilizado Internet en los últimos tres meses», «Ha utilizado Internet en el último año» y «No utiliza Internet». La convención establecida por el Instituto Nacional de Estadística, y que nosotros seguimos en este trabajo, es considerar usuario de Internet a toda aquella persona que, al menos, ha utilizado Internet en los últimos tres meses 5 . Por su parte, para medir los usos de Internet, se propuso a los encuestados una pregunta dicotómica con una batería de 16 ítems que abarcaban una gran variedad de usos comunes de Internet, que van desde enviar correos electrónicos hasta comprar entradas para espectáculos. Así, se ha obtenido una variable numérica que da cuenta de ambos aspectos y cuyos valores varían entre 0 y 64 . Esta variable se ha construido sumando las puntuaciones de cada encuestado en cada una de las preguntas mencionadas y asignando las puntuaciones más altas a aquellas personas que realizan un uso de Internet con las frecuencias más altas (todos los días, una vez a la semana, etc.).

En relación con las variables sociodemográficas, se han utilizado la edad, el nivel de estudios y el estatus socioeconómico. Estas tres variables han sido construidas, literalmente, siguiendo las recomendaciones del Instituto Nacional de Estadística para la ya referida Encuesta sobre equipamiento y uso de tecnologías de información y comunicación en los hogares. La variedad lugares de conexión es una

4. Dado que la población de internautas es infinita, el error muestral de la población de internautas es prácticamente idéntico al de la población en general. Sin embargo, al no tener un marco muestral de los internautas, no podemos ofrecer un valor concreto.

5. Esta decisión queda expuesta en las tablas, en los informes y en el diseño metodológico de las Encuestas sobre Equipamiento y Uso de Tecnologías de Información y Comunicación en los Hogares, producidas por el Instituto Nacional de Estadística (http://www.ine.es/ inebmenu/mnu_tic.htm). 
variable numérica construida sumando el número de lugares desde los cuales el internauta suele acceder a Internet. Sus valores van del 0 al 4 . La variable tipo de conexión a Internet es también numérica y se ha obtenido sumando las puntuaciones de cada sujeto en función del número y de las tipologías de conexiones de las que dispone. Las puntuaciones más altas se han asignado a las conexiones que garantizan movilidad y autonomía en el acceso a Internet, como el 3G y el wifi. Las más bajas se han asignado a las tecnologías que obligan al usuario a ir a un sitio concreto para poder estar conectado. Sus puntuaciones varían entre 0 y 10 .

En relación con las habilidades digitales, se ha construido una variable numérica que recoge las respuestas de las personas a 14 ítems que representan operaciones elementales que se realizan al navegar por Internet (introducir una dirección en la barra de navegación, descargar un fichero, eliminar el historial de navegación, etc.). A cada respuesta positiva, se le asignó un punto. Así, los sujetos pueden obtener puntuaciones que varían entre 0 y 14 .

Finalmente, las variables psicológicas y actitudinales, es decir la facilidad de uso y la utilidad percibida, junto con los valores postmaterialistas, se han construido a través de un conjunto de indicadores ordinales que oscilan entre 1 y 5 . Cada una de estas variables se ha construido sumando las puntuaciones obtenidas en cada uno de los ítems. Se han obtenido así tres escalas con puntuaciones que varían, respectivamente, entre 0 y 20 ; entre 0 y 30 , y entre 0 y 15 .

Para el detalle de las preguntas del cuestionario utilizadas en cada una de las variables construidas, así como los datos técnicos de la encuesta, véase el anexo 1.

\section{Resultados}

En un primer momento, se ha implementado un modelo de path analysis para corroborar el sistema clásico del TAM, que incluye tanto sus variables psicológicas (utilidad percibida y facilidad de uso) como sus variables sociodemográficas más utilizadas en el estudio de la brecha digital (edad, estatus socioeconómico y nivel de estudios). Concretamente, se ha evaluado un conjunto de relaciones que preveían la influencia de las variables sociodemográficas sobre las del TAM, que, a su vez, tuvieran influencia sobre nuestra variable dependiente ${ }^{6}$. Este modelo tiene la siguiente forma (ver figura 1$)^{7}$.

Los índices de ajuste obtenidos con la implementación del análisis no cumplen con los criterios exigidos para la aceptación del modelo (Ruiz et al., 2010).

6. Para implementar el path analysis, ha sido necesario imputar las puntuaciones de todas las variables a los casos perdidos. Para ello, se ha optado por la opción «interpolación lineal en el punto».

7. Este modelo ha sido tomado del trabajo de varios autores de la disciplina, como LópezBonilla y López-Bonilla (2012), Venkatesh y Davis (2000) o Malhotra y Galletta (1999). No hemos incluido en el modelo las actitudes hacia las tecnologías. Tal y como se comentó más arriba, los estudios recientes han mostrado la baja incidencia de esta variable y han dejado de utilizarla de forma sistemática. 
Figura 1. Modelo analítico

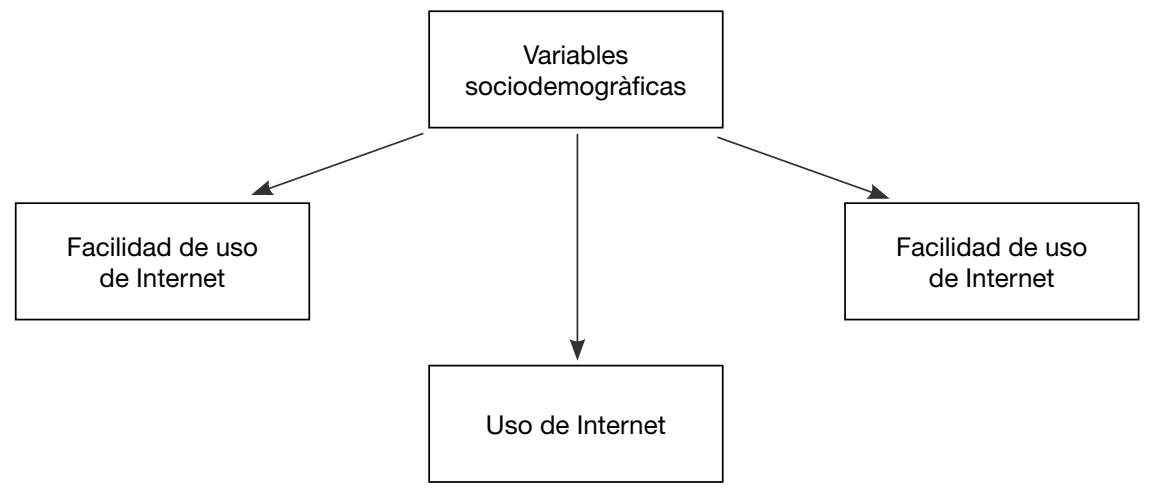

Fuente: elaboración propia.

Consecuentemente, los resultados indican que este modelo no se ajusta a la matriz de datos.

Además, los coeficientes de regresión, presentados en la tabla 2, manifiestan que el estatus socioeconómico no parece mantener ninguna relación significativa con las dos variables psicológicas. El nivel de estudios, por otra parte, solo mantiene una relación significativa con la utilidad percibida, pero no con la facilidad de uso.

En segundo lugar, se ha implementado un modelo de regresión múltiple "por pasos». La tabla 3 pone en evidencia el hecho de que todos los modelos son significativos. Hasta el tercer paso, es decir, hasta la introducción de las

Tabla 1. Índices de ajuste del modelo

\begin{tabular}{lrrr}
\hline Estadístico & & & $\begin{array}{c}\text { Valor } \\
\text { obtenido }\end{array}$ \\
\hline Ajuste comparativo & Abreviatura & Criterio & \\
Índice de bondad de ajuste comparativo & & & \\
Índice de Tucker-Lewis & CFI & $>0,9$ & 0,341 \\
Índice de ajuste normalizado & TLI & $>0,9$ & $-0,41$ \\
Ajuste normalizado & NFI & $>0,9$ & 0,345 \\
NFI corregido por parsimonia & & & \\
\hline Otros & PNFI & Próximo a 1 & 0,161 \\
Índice de bondad de ajuste & & & \\
Índice de bondad de ajuste corregido & GFI & $>0,9$ & 0,84 \\
Raíz del residuo cuadrático medio & AGFI & $>0,9$ & 0,52 \\
Raíz de residuo cuadrático promedio de aproximación & RMR & Próximo a 0 & 4,505 \\
\hline
\end{tabular}

Fuente: elaboración propia a partir de los datos de la encuesta realizada. 
Tabla 2. Coeficientes de regresión

\begin{tabular}{llccc}
\hline & & Estimación & Desviación estándar & $\mathrm{P}$ \\
\hline Utilidad & $\leftarrow$ Edad & $-0,091$ & 0,012 & $* \star \star$ \\
Facilidad & $\leftarrow$ Edad & $-0,035$ & 0,007 & $* \star \star$ \\
Utilidad & $\leftarrow$ Estudios & 0,7 & 0,147 & ${ }^{* \star \star}$ \\
Facilidad & $\leftarrow$ Estudios & 0,114 & 0,083 & 0,169 \\
Utilidad & $\leftarrow$ Estatus & 0,112 & 0,172 & 0,515 \\
Facilidad & $\leftarrow$ Estatus & 0,066 & 0,098 & 0,498 \\
Frecuencia y cantidad de usos & $\leftarrow$ Utilidad & 3,591 & 0,263 & $* \star \star$ \\
Frecuencia y cantidad de usos & $\leftarrow$ Facilidad & 2,101 & 0,477 & $* \star \star$ \\
\hline
\end{tabular}

Fuente: elaboración propia a partir de los datos de la encuesta realizada.

Tabla 3. Resultados de los análisis de regresión múltiple 1

\begin{tabular}{|c|c|c|c|c|c|}
\hline & Modelo & $\begin{array}{c}\text { Suma de } \\
\text { cuadrados }\end{array}$ & GI & Media cuadrática & $\mathrm{F}$ \\
\hline \multirow[t]{3}{*}{1} & Regresión & 32224,690 & 3 & 10741,563 & \\
\hline & Residual & 161237,593 & 1082 & 148,956 & $72,112^{\star \star \star}$ \\
\hline & Total & 193462,283 & 1085 & & \\
\hline \multirow[t]{3}{*}{2} & Regresión & 64500,836 & 5 & 12900,167 & \\
\hline & Residual & 128961,446 & 1080 & 119,359 & $108,079^{\star \star \star}$ \\
\hline & Total & 193462,283 & 1085 & & \\
\hline \multirow[t]{3}{*}{3} & Regresión & 90531,833 & 6 & 15088,639 & \\
\hline & Residual & 102930,450 & 1079 & 95,354 & $158,237^{\star \star \star}$ \\
\hline & Total & 193462,283 & 1085 & & \\
\hline \multirow[t]{3}{*}{4} & Regresión & 97725,716 & 9 & 10858,413 & \\
\hline & Residual & 95736,567 & 1076 & 88,937 & $122,091^{\star \star \star}$ \\
\hline & Total & 193462,283 & 1085 & & \\
\hline
\end{tabular}

Fuente: elaboración propia a partir de los datos de la encuesta realizada.

${ }^{* \star \star} p<0,001 ;{ }^{* \star} p<0,01 ;{ }^{*} p<0,05$.

habilidades digitales, los valores del estadístico F presentan una pauta de crecimiento. Esto implica que los modelos se vuelven más precisos para estimar la variable dependiente a medida que se añaden bloques de variables. Sin embargo, al introducir las variables psicológicas, el valor del estadístico $\mathrm{F}$ disminuye. Así pues, el cuarto modelo resulta ser menos preciso que el tercero en cuanto a predicción.

En la tabla 4, se incluyen los porcentajes de varianza explicada por los diferentes bloques del modelo.

En total, el modelo de regresión implementado da cuenta del 50\% de la varianza de la variable frecuencia y variedad de usos de Internet. El primer bloque, es decir, el de las variables sociodemográficas, explica el 16,6\% de la varianza de la variable dependiente. El segundo bloque, el de las variables contextuales, explica 
Tabla 4. Porcentajes de varianza explicada

\begin{tabular}{ccccc}
\hline Modelo & $\mathbf{R}$ & R cuadrado & R cuadrado corregida & Cambio en R cuadrado \\
\hline 1 & 0,408 & 0,167 & 0,164 & 0,167 \\
2 & 0,577 & 0,333 & 0,330 & 0,167 \\
3 & 0,684 & 0,468 & 0,465 & 0,135 \\
4 & 0,711 & 0,505 & 0,501 & 0,037 \\
\hline
\end{tabular}

Fuente: elaboración propia a partir de los datos de la encuesta realizada.

el 16,7\% de la varianza. Las habilidades digitales explican el 13,6\% de la varianza total. Finalmente, el cuarto bloque, compuesto por las variables del TAM más los valores postmaterialistas, explica solo el 3,6\% de la varianza. Nuevamente, este bloque aporta menos a nuestra capacidad predictiva que los bloques anteriores.

Por último, observando los coeficientes de regresión, destacamos que, entre las variables sociodemográficas, tanto la edad como el nivel de estudios mantienen, en el cuarto modelo, relaciones significativas con la variable dependiente. Esto confirma los resultados de los análisis realizados anteriormente (Torres et al., 2011). Ambas relaciones son positivas. Las dos variables contextuales, variedad de lugares de conexión y calidad de las conexiones, son significativas y mantienen una relación positiva con la variable dependiente. Lo mismo sucede con las habilidades digitales, que se transforman en las variables con mayor peso. Entre las variables psicológicas, son significativas la percepción de utilidady los valores postmaterialistas, pero no la facilidad de uso de la tecnología.

\section{Discusión}

Nuestros análisis empíricos nos ofrecen información relevante para avanzar en la discusión planteada en este artículo. En primer lugar, los resultados que hemos obtenido indican que el modelo clásico del TAM no cumple con los criterios empíricos básicos aceptados por las ciencias sociales (Ruiz et al., 2010). Ciertamente, existe la relación de significatividad entre las variables psicológicas y nuestra variable dependiente. Sin embargo, el modelo no permite establecer la existencia de una relación entre todas las variables sociodemográficas y las variables psicológicas, tal y como pronosticaba el modelo. Sí existe, en cambio, una relación entre la edad, la facilidad de uso y la utilidad percibida. Pese a ello, en general, y tomando como referentes los resultados del path analysis, no puede concluirse que las relaciones entre las variables implicadas en el modelo clásico del TAM se comporten de la forma esperada.

Nuestra segunda estrategia de análisis ofrece más información relevante. En primer lugar, debe destacarse que nuestro segundo modelo permite predecir el $50 \%$ de la varianza de la variable dependiente. Estamos, pues, ante un modelo con gran capacidad predictiva. Sin embargo, se observa también, a través del cambio en el R cuadrado, que el grupo de variables que más capacidad predictiva suma a nuestro modelo son los compuestos por las variables contextuales y sociodemográficas. Sin embargo, al añadir a nuestro modelo las variables 
Tabla 5. Resultados de los análisis de regresión múltiple 2

\begin{tabular}{|c|c|c|c|c|}
\hline & Modelos & B & Error típ. & Beta \\
\hline \multirow[t]{4}{*}{1} & Constante & 10,097 & 2,456 & \\
\hline & Edad & $-0,241$ & 0,028 & $-0,237^{\star \star \star}$ \\
\hline & Estudios & 3,777 & 0,400 & $0,298^{* * *}$ \\
\hline & Estatus & 1,622 & 0,465 & $0,110^{* \star}$ \\
\hline \multirow[t]{6}{*}{2} & Constante & $-3,595$ & 2,354 & \\
\hline & Edad & $-0,033$ & 0,028 & $-0,032$ \\
\hline & Estudios & 2,737 & 0,364 & $0,216^{\star \star \star}$ \\
\hline & Estatus & 0,569 & 0,422 & 0,038 \\
\hline & Calidad conexiones & 1,242 & 0,131 & $0,256^{\star \star \star}$ \\
\hline & Cantidad conexiones & 4,270 & 0,392 & $0,315^{\star \star \star}$ \\
\hline \multirow[t]{7}{*}{3} & Constante & $-13,427$ & 2,186 & \\
\hline & Edad & 0,050 & 0,026 & 0,049 \\
\hline & Estudios & 1,777 & 0,331 & $0,14^{\star \star \star}$ \\
\hline & Estatus & 0,399 & 0,377 & 0,027 \\
\hline & Calidad conexiones & 0,745 & 0,121 & $0,153^{\star \star \star}$ \\
\hline & Cantidad conexiones & 2,961 & 0,359 & $0,218^{\star \star \star}$ \\
\hline & Habilidades & 2,133 & 0,129 & $0,438^{* \star *}$ \\
\hline \multirow[t]{10}{*}{4} & Constante & $-26,577$ & 2,816 & \\
\hline & Edad & 0,066 & 0,025 & $0,065^{\star \star}$ \\
\hline & Estudios & 1,677 & 0,320 & $0,132^{* * *}$ \\
\hline & Estatus & 0,498 & 0,364 & 0,034 \\
\hline & Calidad conexiones & 0,619 & 0,118 & $0,127^{\star \star \star}$ \\
\hline & Cantidad conexiones & 2,581 & 0,350 & $0,190^{\star \star \star}$ \\
\hline & Habilidades & 1,918 & 0,128 & $0,391^{\star \star \star}$ \\
\hline & Facilidad & 0,189 & 0,112 & 0,041 \\
\hline & Utilidad & 0,455 & 0,066 & $0,175^{\star \star \star}$ \\
\hline & Postmaterialismo & 0,188 & 0,092 & $0,045^{\star}$ \\
\hline
\end{tabular}

Fuente: elaboración propia a partir de los datos de la encuesta realizada.

${ }^{* \star *} p<0,001 ;{ }^{* \star} p<0,01 ;{ }^{*} p<0,05$.

psicológicas, solo se aumenta en un 3,7\% la capacidad predictiva. Esto indica el peso relativo de cada uno de los factores explicativos utilizados en este análisis. Por otra parte, se observa, gracias a los coeficientes de regresión, que únicamente la utilidad percibida mantiene, en el cuarto paso de nuestro modelo, una relación significativa con la variable dependiente. Sin embargo, la edad, el nivel de estudios, los valores postmaterialistas, la calidad de las conexiones, la cantidad de las conexiones y, especialmente, las habilidades digitales, sí muestran una relación significativa con el uso de Internet.

Esto obliga a reflexionar sobre, en general, la estructura del modelo TAM y, en particular, sobre el propio mecanismo tras las motivaciones para comenzar a utilizar Internet. Ahora sabemos que, tras el comportamiento observado en este trabajo, se encuentran procesos individuales y psicológicos, pero que estos 
obedecen a patrones de interacción social, a valores sociales emergentes y a la propia relación con Internet (habilidades digitales). Mientras el mecanismo explicativo tradicional ponderaba la dimensión psicológica del uso de Internet, nuestro trabajo completa y mejora la compresión de los procesos tras este tipo de comportamiento.

El modelo TAM sociológico que defendemos aquí estaría formado, por una parte, por una de las variables psicológicas originales del TAM, la utilidad percibida del uso de Internet. Es decir, cuanta mayor es la percepción de la utilidad de esta tecnología, mayor es la variedad de usos dados a Internet, así como el tiempo dedicado a la red. Sin embargo, nuestro análisis obliga a completar el modelo con variables que se han mostrado significativas y que se refieren a recursos individuales (habilidades digitales, nivel de estudios y edad), a recursos materiales con los que cuentan los internautas y a los valores que, de acuerdo con Inglehart (1991), comienzan a ser socialmente hegemónicos: los valores postmaterialistas. Por lo tanto, el TAM sociológico es un modelo en el que se contemplan el contexto, las características y los valores sociales del internauta, así como la percepción sobre las posibilidades del medio. Nuestro modelo, por tanto, pone al mismo nivel lo social y lo tecnológico, y trata de mostrar las relaciones entre estos dos niveles evitando los efectos del determinismo tecnológico y social.

Desde nuestro punto de vista, para comprender adecuadamente estos resultados, es necesario ponerlos en contexto. Tal y como hemos señalado más arriba, la relación entre tecnología y sociedad no es estática. La percepción sobre Internet, así como las formas y el grado en las que esta tecnología es utilizada, varían a lo largo del tiempo. Los resultados de la aplicación del modelo TAM al uso de Internet mostraron la relevancia de variables como la utilidad percibida del uso de Internet y la facilidad de uso de la tecnología. Sin embargo, estas variables, especialmente la segunda de ellas, son menos relevantes a medida que la tecnología es más conocida y su uso se extiende. Así, la importancia de esta variable va perdiendo protagonismo en favor de las habilidades digitales. En una sociedad en la que Internet tiene cada vez mayor presencia y en la que la penetración de esta tecnología se extiende a prácticamente todos los ámbitos, la cuestión no es tanto si dicha tecnología es fácil o difícil de usar. El porcentaje de usuarios de Internet no ha dejado de aumentar a lo largo de la última década y, con ello, también ha crecido el número de ciudadanos que perciben Internet como una herramienta suficientemente fácil de usar como para realizar, al menos, actividades de carácter básico. Sin embargo, esto no sucede con las habilidades digitales. Estas requieren formación y experiencia. Por tanto, la cuestión no es tanto si los ciudadanos consideran Internet fácil o difícil de usar, sino qué grado de pericia digital tienen y qué actividades pueden realizar con esos recursos. Esta dimensión contextual del uso de Internet es, como hemos mostrado en el presente trabajo, básica para mejorar la capacidad predictiva del modelo TAM.

Desde un punto de vista dinámico, es esperable que la variable edad pierda protagonismo en los análisis sobre el uso de Internet, en la medida en que el 
tiempo transforme a los jóvenes de hoy en adultos o mayores de mañana. Sin embargo, es relevante observar la importancia de la variable nivel de estudios. Pese a que el uso de Internet ha avanzado entre los ciudadanos menos formados, la educación aún continúa siendo clave. Sabemos que, cuanto más alto es el nivel de estudios, mayor es la probabilidad de ser un usuario de Internet avanzado. Así, es fundamental que esta variable forme parte del modelo, ya que, sin su presencia, sería imposible medir hasta qué punto las desigualdades sociales tienen un efecto sobre nuestro objeto de análisis. Nuevamente, la inclusión de una variable social nos permite mejorar no solo la capacidad predictiva del modelo, sino también su dimensión analítica.

Lo mismo sucede con las variables que miden las condiciones materiales de conexión a Internet. El desarrollo de la red genera que los recursos técnicos sean fundamentales para mejorar la capacidad de los internautas para obtener más beneficios de este medio. Contar con una tecnología actualizada se ha convertido, así, en un factor clave para transformarse en un usuario avanzado. Nuevamente, la inclusión de esta variable, que el análisis ha mostrado relevante, nos permite mejorar la explicación sustantiva.

Nuestro análisis nos ha permitido mejorar la capacidad predictiva del modelo TAM clásico, así como complementarlo con variables que ofrecen un perfil marcadamente sociológico. La constatación de que el modelo TAM sociológico es un mejor predictor del uso de Internet permite contrastar la hipótesis que nos planteamos al comienzo del artículo. Sin embargo, también debe destacarse la importancia de vincular una explicación analítica e individualista metodológica, como la que presentamos aquí, con procesos más amplios y generales, como el cambio tecnológico. Estamos convencidos de que únicamente a través de un vínculo entre estos dos niveles analíticos es posible establecer conclusiones explicativas sobre este segmento de la realidad social que nos ha ocupado. Este ha sido el objetivo final de nuestro trabajo.

\section{Conclusión}

A lo largo de los últimos años, en nuestra tarea investigadora, hemos prestado especial atención al papel del modelo TAM para explicar el uso de la tecnología, muy especialmente de las TIC en general y de Internet en particular. Aunque era un modelo bien conocido en otras áreas de las ciencias sociales de lengua española, como la gestión de la innovación o los estudios organizacionales (López-Bonilla y López-Bonilla, 2006), se trataba de un modelo escasamente conocido en la comunidad sociológica, seguramente debido a que su formulación clásica y habitual se ha basado, de una manera muy acentuada, en variables psicológicas y en un enfoque de corte individualista. No obstante, el modelo siempre se ha caracterizado por la rigurosidad de sus análisis empíricos y de sus propuestas teóricas, así como por su capacidad para su propia evolución teorética, lo que ha supuesto una perceptible decantación a interesarse por el papel de algunos factores vinculados al contexto social en el que los individuos toman sus decisiones. Todas estas razones explican el adelanto 
y el interés previo de los colegas de algunas de las vecinas áreas de problemas de la sociología, pero también su dificultad para una integración fértil de las variables sociales.

Este artículo conforma el tercer eslabón de ese decidido giro sociológico que pretendemos dar con el modelo TAM. El primero (Torres et al., 2011) ponía de manifiesto, a partir de la evidencia empírica de una encuesta representativa de la población española, el mayor peso de las variables de la edad y el nivel de estudios para dar cuenta del uso de Internet, frente a las variables clásicas de la utilidad percibida del uso de Internet y la facilidad de uso de la tecnología. Los ya referidos sólidos comentarios de nuestros críticos nos permitieron realizar una réplica y, con ello, un segundo eslabón (Torres et al., 2012), en el que afianzamos argumentalmente el giro sociológico del TAM sin tener que renunciar a una estrategia multidisciplinaria que no acotase la fuerza de lo social a un enfoque psicológico e individualista. Finalmente, una segunda encuesta representativa de la población española nos ha permitido afinar esta orientación social realizada desde la sociología, al incorporar un haz mayor de variables independientes (sociodemográficas, contextuales, de habilidades y de valores), así como de operacionalizar de manera más sofisticada la dependiente (acerca del uso de Internet). El resultado, a nuestro juicio, es una mayor robustez del giro sociológico que se concreta en un modelo plural en el que destacan los recursos individuales (utilidad de la tecnología y de las habilidades digitales) junto a las variables psicosociales (valores postmateriales), contextuales (cantidad de lugares de conexión y calidad de las conexiones) y sociales (nivel de estudios y edad). La fuerza del modelo sociológico se expresa en que la creciente extensión de la red permite apreciar cómo las variables sociales no solo ejercen su papel de impulsoras (normalización), sino también, y sobre todo, de limitadoras (estratificación) en el uso universal de Internet.

\section{Referencias bibliográficas}

Aibar, Eduardo (1996). «La vida social de las máquinas: Orígenes, desarrollo y perspectivas actuales en la sociología de la tecnología». Reis [en línea], 70, 141-170. <http://dx.doi.org/10.2307/40183990>.

Davis, Fred D. (1986). A technology acceptance model for empirically testing new end-user information systems: Theory and results. Cambridge: Massachusetts Institute of Technology.

- (1989). «Perceived usefulness, perceived ease of use, and user acceptance of information technology». MIS Quarterly [en línea], 13 (3), 319-340. $<$ http://dx.doi.org/10.2307/249008>.

Davis, Fred D.; Bagozzi, Richard P. y Warshaw, Paul R. (1989). «User acceptance of computer technology: A comparison of two theoretical models». Management Science [en línea], 35 (8), 982-1003. $<$ http://dx.doi.org/10.1287/mnsc.35.8.982>.

- (1992). «Extrinsic and intrinsic motivation to use computers in the workplace». Journal of Applied Social Psychology [en línea], 22 (14), 1111-1132. $<$ http://dx.doi.org/10.1111/j.1559-1816.1992.tb00945.x>. 
DiMaggio, Paul; Hargittai, Eszter; Neuman, W. Russell y Robinson, John P. (2001). «Social implications of the Internet». Annual Review of Sociology [en línea], 27 (1), 307-336. <http://dx.doi.org/10.1146/annurev.soc.27.1.307>.

ElSTER, Jon (1990). El cambio tecnológico: Investigaciones sobre la racionalidad y la transformación social. Barcelona: Gedisa.

Gómez, Mikel; Sánchez, Miguel y Puerta, Enrique de la (1992). El cambio tecnológico hacia el nuevo milenio: Debates y nuevas teorias. Barcelona: Icaria.

Hargittai, Eszter (2008). "Whose Space?: Differences Among Users and Non-Users of Social Network Sites». Journal of Computer-Mediated Communication [en línea], 1, 276-297. <http://dx.doi.org/10.1111/j.1083-6101.2007.00396.x>.

- (2010). «Digital Na(t)ives?: Variation in Internet Skills and Uses among Members of the "Net Generation"». Sociological Inquiry [en línea], 80, 92-113. <http://dx.doi.org/10.1111/j.1475-682x.2009.00317.x>.

INGLEHART, Ronald (1991). El cambio cultural en las sociedades industriales avanzadas. Madrid: CIS.

Levy, Pierre (2007). Cibercultura: La cultura de la sociedad digital. Barcelona: Anthropos.

Lin, Chieh-Peng y Bhattacherjee, Anol (2009). "Understanding online social support and its antecedents: A socio-cognitive model». The Social Science Journal [en línea], 46 (4), 724-737. <http://dx.doi.org/10.1016/j.soscij.2009.03.004>.

López-Bonilla, Jesús Manuel y López-Bonilla, Luis Miguel (2006). «Estudio comparado de las estimaciones de dos versiones del modelo de aceptación de la tecnología (TAM) mediante los programas Amos y Pls». Revista de Investigaciones Europeas de Dirección y Economía de la Empresa, 12 (3), 95-110.

- (2012). «Perspectiva socio-psicológica del modelo de aceptación de la tecnología: Revisión crítica del trabajo de Torres, Robles y Molina». Revista Internacional de Sociología [en línea], 70, 205-211. <http://dx.doi.org/10.3989/ris.2011.05.13>.

LynotT, Patricia Passuth y McCandless, N. Jane (2000). «The impact of age vs. life experience on the gender role attitudes of women in different cohorts». Journal of Women \& Aging [en línea], 12 (1-2), 5-21. <http://dx.doi.org/10.1300/j074v12n01_02>.

MacKenzie, Donald y Wajcman, Judy (eds.) (1999). The Social Shaping of Technology. Buckingham: Open University Press.

Malmotra, Yogesh y Galletta, Dennis F. (1999). Extending the technology acceptance model to account for social influence: Theoretical bases and empirical validation [en línea]. Documento de trabajo para la 32 edición del Encuentro sobre Sistemas Científicos celebrado en Hawai en 1999. <http://dx.doi.org/10.1109/hicss.1999.772658>.

Mathieson, Kieran (1991). «Predicting user intentions: Comparing the technology acceptance model with the theory of planned behavior». Information Systems Research [en línea], 2 (3), 173-191. $<$ http://dx.doi.org/10.1287/isre.2.3.173>.

Morris, Michael G. y Venkatesh, Viswanath (2000). «Age differences in technology adoption decisions: Implications for a changing work force». Personnel Psychology [en línea], 53 (2), 375-403.

<http://dx.doi.org/10.1111/j.1744-6570.2000.tb00206.x>. 
Noguera, José A. (ed.) (2010). Teoría sociológica analítica. Madrid: CIS.

Norris, Pippa (2001). Digital divide: Civic engagement, information poverty, and the Internet worldwide [en línea]. Cambridge: Cambridge University Press. <http://dx.doi.org/10.1017/CBO9781139164887>.

Quintanilla, Miguel Ángel (2005). Tecnologia: Un enfoque filosófico y otros ensayos de filosofia de la tecnología. México: Fondo de Cultura Económica.

Robles, José Manuel; Torres, Cristóbal y Molina, Óscar (2010). «La brecha digital: Un análisis de las desigualdades tecnológicas en España». Sistema, 218, 3-22.

Robles, José Manuel y Torres, Cristóbal (2012). «Digital Divide and the Information and Communication Society in Spain». Sociologija i Prostor [en línea], 194 (3), 291-307. <http://dx.doi.org/10.5673/sip.50.3.1>.

Ruiz, Miguel A.; PARDo, Antonio y San Martín, Rafael (2010). «Modelos de ecuaciones estructurales». Papeles del Psicólogo, 31 (1), 34-45.

TAYLOR, Shirley y TODD, Peter A. (1995). «Understanding information technology usage: A test of competing models». Information Systems Research [en línea], 6 (2), $144-176$. <http://dx.doi.org/10.1287/isre.6.2.144>.

Torres, Cristóbal; Robles, José Manuel y De Marco, Stefano (2013). «Digital Divide and Digital Inequality in Europe: An Analytic Approach». En: López Peláez, Antonio (ed.). The Robotic Divide: A New Frontier in the XXI Century? [en línea]. Londres: Springer-Verlag. <http://dx.doi.org/10.1007/978-1-4471-5358-0_10>.

Torres, Cristóbal; Robles, José Manuel y Molina, Óscar (2011). «¿Por qué usamos las tecnologías de la información y las comunicaciones?: Un estudio sobre las bases sociales de la utilidad individual de Internet». Revista Internacional de Sociología [en línea], 69, 371-392. $<$ http://dx.doi.org/10.3989/ris.2010.01.15>.

- (2012). «Una inyección sociológica al modelo de la aceptación de la tecnología. Respuesta a nuestros críticos». Revista Internacional de Sociología [en línea], 70.1, 212-216. $<$ http://dx.doi.org/10.3989/ris.2011.05.13>.

VAN DijK, Jan A.G.M. (2005). The deepening divide: Inequality in the information society [en línea]. Los Ángeles: Sage Publications. $<$ http://dx.doi.org/10.4135/9781452229812>.

VenKatesh, Viswanath y Davis, Fred D. (2000). «A theoretical extension of the technology acceptance model: Four longitudinal field studies». Management Science [en línea], 46 (2), 186-204. <http://dx.doi.org/10.1287/mnsc.46.2.186.11926>.

Venkatesh, Viswanath; Morris, Michael G.; Davis, Gordon B. y Davis, Fred D. (2003). «User acceptance of information technology: Toward a unified view». MIS Quarterly, 27 (3), 425-478. 


\section{Anexo 1}

\section{Ficha técnica}

Ámbito: nacional.

Universo: población española de ambos sexos de 18 años y más.

Tipo de encuesta: encuesta modalidad CATI.

Tamaño de la muestra: 1.526 entrevistas.

Afijación: proporcional.

Ponderación: no procede.

Procedimiento de muestreo: las 1.526 entrevistas estratificadas por la intersección hábitat/comunidad autónoma y distribuidas de manera proporcional al total de la región. Se aplicaron cuotas de sexo y edad a la unidad última (persona entrevistada).

Error muestral: partiendo de los criterios del muestreo aleatorio simple, para un nivel de confianza del 95,5\% (que es el habitualmente adoptado) y en la hipótesis más desfavorable de máxima indeterminación $(p=q=50)$, el margen de error de los datos referidos al total de la muestra es de $\pm 2,6$.

Fecha de realización: del 4 al 14 de junio de 2013.

Preguntas para la construcción de la variable dependiente

P.1. ¿Cuándo fue la última vez que utilizó Internet?

1 En el último mes 1

2 Hace más de un mes y menos de tres meses 2

3 Hace más de tres meses y menos de un año 3

4 Hace más de un año 4

5 No lo he utilizado nuca 5

\section{P.5. Digame ahora si, en estos tres últimos meses, ha usado o no ha usado} Internet para realizar alguna de las siguientes actividades:

\section{Sí No}

1 Recibir o enviar correos electrónicos.

2 Participar en redes sociales (Facebook, Twitter, etc.).

3 Leer o descargar noticias, periódicos o revistas de actualidad en línea.

4 Buscar información sobre temas de salud (p. ej.: lesiones, enfermedades, nutrición, etc.).

5 Buscar información sobre educación, formación u otro tipo de cursos.

6 Buscar información sobre bienes y servicios.

7 Consultar wikis (como Wikipedia) o enciclopedias en línea para obtener conocimientos.

8 Buscar mapas u otra información geográfica.

9 Telefonear o realizar videollamadas a través de Internet. 
10 Usar la banca electrónica.

11 Comprar películas y/o música.

12 Comprar libros, revistas, periódicos (incluidos libros electrónicos).

13 Comprar ropa.

14 Comprar billetes de medios de transporte (avión, tren, etc.).

15 Alquilar alojamiento de vacaciones (hotel, apartamento, etc.).

16 Comprar entradas para espectáculos (cine, conciertos, etc.).

\section{Preguntas para la construcción de la variable habilidades digitales}

P.6. A la hora de navegar por Internet, se presentan muchas alternativas posibles. Entre las siguientes que le voy a leer, dígame las que ha realizado o no al menos en alguna ocasión en los últimos tres meses.

\section{Sí No}

1 Abrir el navegador (p. ej.: Explorer o Mozilla).

2 Escribir la dirección de una página web en la barra del navegador.

3 Pinchar en un enlace a una página web.

4 Eliminar el historial de navegación.

5 Abrir una página web nueva en otra ventana, sin cerrar la anterior.

6 Abrir archivos (de textos, imágenes o vídeos) disponibles en línea.

7 Emplear motores de búsqueda (p. ej.: Google) para encontrar información de interés.

8 Acotar la búsqueda de información a una frase o a una expresión exacta.

9 Acotar la búsqueda de información a formatos concretos de archivos (p. ej.: textos, imágenes o vídeos).

10 Guardar, en mi ordenador o en cualquier dispositivo personal, una copia cumplimentada de un formulario en línea.

11 Adjuntar archivos a correos electrónicos.

12 Mantener un blog.

13 Crear una página web.

14 Programar en HTML.

Preguntas para la construcción de la variable actitudes hacia la tecnología

P.9. Con independencia de si usted usa o no usa Internet, jen qué medida está usted de acuerdo o en desacuerdo con cada una de las siguientes afirmaciones que le voy a leer? Para ello, vamos a usar una escala entre 1 y 5, en la que el 1 significa que usted está muy en desacuerdo y el 5, que está muy de acuerdo. Por supuesto, puede usar cualquier puntuación entre ambos extremos.

1 Aprender a usar Internet es fácil.

2 Me siento cómodo navegando por Internet, ya que se adapta a mis capacidades y habilidades.

3 En Internet, es sencillo encontrar lo que busco o lo que necesito.

4 Internet es algo que está siempre a mano y disponible. 
P.10. Y en este mismo orden de cosas, ¿podría decirme en qué medida Internet mejora o podría mejorar cada uno de los siguientes aspectos de su vida que le voy a leer? Vamos a volver a usar la escala entre 1 y 5 , en la que el 1 significa que usted está muy en desacuerdo y el 5, que está muy de acuerdo. Por supuesto, puede usar cualquier puntuación entre ambos extremos.

1 Las actividades laborales y profesionales.

2 Las oportunidades de ocio.

3 Las posibilidades de consumo.

4 Las gestiones bancarias u otras actividades económicas.

5 El contacto con familiares, amigos o conocidos.

6 La información y el conocimiento sobre temas de interés.

P.11. Voy a leerle un conjunto de afirmaciones. Para cada una de ellas, dígame en qué medida está usted de acuerdo o en desacuerdo. Para ello, volvemos a usar la escala entre 1 y 5, en la que el 1 significa que usted está muy en desacuerdo y el 5, que está muy de acuerdo. Por supuesto, puede usar cualquier puntuación entre ambos extremos.

1 Internet refuerza los vínculos sociales y hace sentirse parte de la comunidad.

2 Internet puede mejorar la capacidad para influir sobre el poder.

3 Internet es fundamentalmente un medio de entretenimiento.

4 Internet ayuda a los ciudadanos a alcanzar sus objetivos en la vida.

5 El mundo es mejor gracias a Internet.

6 Internet es, fundamentalmente, un medio para estar en contacto con los amigos y familiares.

\section{Preguntas para la construcción de las variables sociodemográficas} y de equipamiento tecnológico

Las variables sociodemográficas y de equipamiento tecnológico han sido tomadas directamente de la Encuesta sobre equipamiento y uso de tecnologías de información y comunicación en los hogares, realizada por el Instituto Nacional de Estadística.

Para más información: http://www.ine.es/dyngs/INEbase/es/operacion.htm? $=$ Estadistica_C\&cid=1254736176741\&menu=ultiDatos\&i $\mathrm{dp}=1254735976608$ 